\title{
Biased interpretations of ambiguous bodily threat information in adolescents with chronic pain
}

Heathcote, L.C. ${ }^{1,2}$, Jacobs, K. ${ }^{3}$, Eccleston, C. ${ }^{4,5}$, Fox, E. ${ }^{1}$, \& Lau, J. Y. F ${ }^{6 *}$.

1 Department of Experimental Psychology, University of Oxford, United Kingdom

2 Department of Anesthesiology, Pain, and Perioperative Medicine, Stanford University, United States

3 Oxford Centre for Children and Young People in Pain (OxCCYP), Nuffield Orthopaedic Centre, United Kingdom

4 Centre for Pain Research, University of Bath, United Kingdom

5 Department of Clinical and Health Psychology, Ghent University, Belgium

6 Department of Psychology, King's College London, United Kingdom

*Corresponding author: Jennifer Lau, Department of Psychology, Institute of Psychiatry, Psychology, and Neuroscience, King's College London, 16 De Crespigny Park, London, SE58AF, United Kingdom. Tel: +44 (0) 207848 0002. Electronic mail may be sent to jennifer.lau@kcl.ac.uk.

Lauren Heathcote is a DPhil student at the University of Oxford, a Research Training Fellow with Action Medical Research for Children (Grant Reference: GN2122), and a trainee member of Pain in Child Health, a research training initiative of the Canadian Institutes of Health Research. Elaine Fox is supported by an ERC Advanced Investigator Award (Ref: 324176).

Category: Original article

Number of text pages: 30

Number of tables: 1

Number of figures: 1

Keywords: interpretation bias, adolescents, chronic pain, cognitive bias, ambiguous situations 


\section{ABSTRACT}

Adult chronic pain patients are consistently shown to interpret ambiguous health and bodily information in a pain-related and threatening way. This interpretation bias may play a role in the development and maintenance of pain and disability. However, no studies have yet investigated the role of interpretation bias in adolescent pain patients, despite that pain often first becomes chronic in youth. We administered the Adolescent Interpretations of Bodily Threat (AIBT) task to adolescents with chronic pain $(N=66)$ and adolescents without chronic pain $(N=74)$. Adolescents were 10-18 years old and completed the study procedures either at the clinic (patient group) or at school (control group). We found that adolescents with chronic pain were less likely to endorse benign interpretations of ambiguous pain and bodily-threat information than adolescents without chronic pain, particularly when reporting on the strength of belief in those interpretations being true. These differences between patients and controls were not evident for ambiguous social situations, and they could not be explained by differences in anxious or depressive symptoms. Further, this interpretation pattern was associated with increased levels of disability among adolescent patients, even after controlling for severity of chronic pain and pain catastrophizing. The current findings extend our understanding of the role and nature of cognition in adolescent pain, and provide justification for employing the AIBT task in longitudinal and training studies to further investigate causal associations between interpretation bias and chronic pain. 


\section{Introduction}

Cognitive-affective models implicate biased cognitive processes in the chronicity and maintenance of pain and pain-related disability $[14,17,18,49,59]$. Whilst evidence for biased attentional processes is mixed [13,54], studies of interpretation bias have demonstrated robust results [53]. Adult chronic pain patients are consistently shown to interpret ambiguous health and bodily information in a pain-related and threatening way [19,30,37,50,51]. However, no studies have yet investigated biased interpretations in youth with chronic pain. As studies of altered cognition in adult chronic pain are likely to be confounded by recurrent episodes of pain and its management, the same studies conducted in youth with chronic pain may be more informative of the role of interpretation bias in earlier episodes of pain, when pain first becomes chronic and disabling [31,48].

To address this gap, we recently developed a new measure to investigate interpretations of ambiguous pain and bodily-threat information in youth: the Adolescent Interpretations of Bodily Threat (AIBT) task [22]. In a community adolescent sample [22], we showed that the tendency to endorse negative interpretations and to reject benign interpretations of ambiguous vignettes was associated with higher pain catastrophizing and recently experienced pain. This interpretation pattern was not specific for situations regarding pain and bodily threat, but generalized across social situations. This lack of specificity may be because the sample was unselected, and we may expect more specific interpretation biases for bodily threat information in clinical pain samples. It is also unknown whether the tendency to interpret ambiguous information as being indicative of pain and bodily threat is clinically-relevant, that is, associated with poorer functioning in pediatric chronic pain patients, as cognitive-affective models would predict. 
In this paper we examine the presence and role of an interpretation bias in adolescents with and without chronic pain. We expected that adolescents with chronic pain would endorse more negative, and less benign interpretations of ambiguous pain and bodily-threat information compared with a control sample of adolescents without chronic pain. We also expected that, unlike in community samples, adolescent patients' bias would not extend to ambiguous social situations. Moreover, given that previous research has shown strong associations between negative interpretation biases and anxious as well as depressive symptoms in adolescents $[5,10,21,45,46,58]$, and that young pain patients often report comorbid psychopathology, we explored whether differences between patients and controls would be due to differences in anxious and depressive symptoms. The AIBT task also allows us to assess whether a negative bias is strongest for when patients report whether interpretations come to mind (interpretation generation) or how strongly they believe the interpretations to be true (interpretation belief), informing the point in the appraisal process that biases are most influential. If differences between adolescents with and without chronic pain were found in any AIBT task indices, we then examined whether these indices are linearly associated with functional disability among adolescents with chronic pain, beyond what is explained by severity of chronic pain, and by another important cognitive factor, pain catastrophizing.

\section{Methods}

\subsection{Participants}

\subsubsection{Patient group}

Adolescents with chronic non-cancer pain were recruited from the Oxford Centre for Children and Young People in Pain (OxCYPP), based at the Nuffield Orthopaedic Centre, part of the 
Oxford University Hospitals. OxCYPP is part of an orthopaedic musculoskeletal referral system, receiving referrals from gastroenterology, neurology, orthopaedics, spinal units, rheumatology, and General Practitioners. Participants were eligible for the current study if they reported recurrent or persistent pain for more than 3 months [38]. Participants were also required to be between ages 10 and 18 years, and fluent in English. Participants were excluded if they were currently experiencing severe distress, based on expert clinician judgment by a consultant clinical psychologist. The recruitment period was between October 2014 and April 2016. Participants were recruited as part of a larger study investigating Attention Bias Modification (ABM) training (data not yet published), but which involved a baseline phase where we also collected data on interpretation bias. Only data purporting to interpretation bias is reported here. As interpretation biases were measured before training, ABM training procedures do not affect data in the current study. Patients and a parent/guardian were first approached about the study either following their first assessment session at the clinic, or following their second visit to the clinic during which they attended a pain education class with a small group of other patients and families. During these sessions, patients and their families were approached by a paediatric rheumatologist or the team's consultant clinical psychologist and asked if they would like to learn more about taking part in a research study. The clinician took verbal consent that the patient and parent/guardian were happy to be approached by a member of the research team, who subsequently provided the information sheet, answered questions about the study, and scheduled appointments. Seventy-three adolescents and their families were interested in the study, and 67 families agreed to take part. The first participant was recruited as a pilot participant and only completed a small number of measures to examine feasibility of testing in the hospital setting. Thus the final sample comprised 66 adolescent participants (55 female; $M=13.97$ years; $S D$ $=2.13$, age range $=10-18$ years). Parents/guardians provided informed consent for their 
children. In addition, participants aged 16 or over provided informed consent for themselves. Participants younger than 16 years provided informed assent. The National Research Ethics Service (NRES), part of the National Health Service (NHS), approved data collection from this patient sample.

\subsubsection{Control group}

Adolescents in the control group were recruited from two secondary schools in the south of England. The recruitment period was between January and July 2015. Members of the research team contacted schools, and the principals gave verbal consent to make contact with adolescents as potential participants. Teachers of eight school classes then invited all pupils of those classes to take part. Parents of the participants were informed about the study by email, and provided informed consent for their children. In addition, participants aged 16 or over provided informed consent for themselves. Participants younger than 16 years provided informed assent. All participants were fluent in English. These participants were recruited and completed the AIBT task for a previous study to examine associations between interpretation bias and acute pain experiences in a community sample. The data from this sample is published elsewhere [22]. For the purposes of the current study, we selected the data from participants in the community sample who did not meet criteria for chronic pain (i.e., those who reported that they had not experienced continuous or recurrent pain for longer than three months). Thus, data from 74 participants was selected to be included in the control group for the current study (42 female; $M=14.95$ years; $S D=1.71$, age range $=11-18$ years). The Central University Research Ethics Committee at the University of Oxford approved data collection from this community sample.

\subsection{Measures}


We collected demographic information to characterise age and gender for both samples. We also collected additional information on demographics and clinical history from the adolescent chronic pain patients to appropriately characterise this sample and allow comparability with other clinical samples in the previous literature. Participants in both samples completed the AIBT task as well as measures of anxiety and depression, and catastrophizing. Only the patients completed a measure of functional disability.

\subsubsection{Adolescent Interpretations of Bodily Threat (AIBT) task}

The Adolescent Interpretations of Bodily Threat (AIBT) task is a computerized measure of interpretation bias for adolescents [22]. The task consists of 16 vignettes describing ambiguous situations. Half of the vignettes describe ambiguous situations that may be interpreted as relating to bodily threat or pain, and half describe ambiguous social situations. The situations reflect events that may occur at school, at home, or during everyday adolescent life, including both self-referential and other-oriented items. In the task, participants are first presented with one of the ambiguous situations in the centre of the screen. An example of a bodily threat situation is as follows: "Your dad jumps out of his chair and puts his hands to his face, making a loud noise. He is....". The situation is ambiguous because there are at least two different possible word endings, reflecting different interpretations. The participants are instructed to first read the situation and to imagine themselves in the situation before pressing the spacebar. After pressing the spacebar, participants are offered one possible end word that resolves the situation in a negative or benign manner. For example, "Your dad jumps out of his chair and puts his hands to his face, making a loud noise. He is hurt". Participants then rate whether that interpretation pops into their mind on a scale of 1 to $5(1=$ doesn't pop into my mind, 3 = might pop into my mind, 5 = definitely pops into my mind). After rating the first word, they are presented with a second word that resolves the situation in a different 
way; for example, "Your dad jumps out of his chair and puts his hands to his face, making a loud noise. He is surprised", and are again asked to rate if that interpretation pops into their mind. Finally, participants are asked to select the interpretation that most easily came to their mind. An example of a social situation is as follows: "You receive a notification that one of your classmates has put a comment on your picture on Facebook. While opening the webpage you think that it will be something nice/nasty." After the participants have responded to all 16 scenarios, they receive new instructions. Participants are informed that they will see the same situations again, however this time, they are asked to rate their belief that each interpretation would actually be happening in that situation $(1=$ not likely, $3=$ maybe likely, $5=$ very likely). The addition of this belief question has been used in a number of previous studies to measure interpretation bias in adults and children (e.g., $[9,43,55])$. Bodily threat and social items are presented in a random order that is fixed between participants. Interpretations (i.e., words ending the sentence) are also presented in a fixed random order so that all participants viewed the same order of items and response choices. Participants were not able to go back to previous items after making a response. Participants were not given a time constraint to complete the AIBT task items.

The current format of the AIBT task, specifically in which participants rate experimentergenerated interpretations rather than generating their own interpretations, was selected to be comparable with similar tools used in previous studies with adolescent populations (e.g., [39]). This format also eliminates the need for experimenters to code participant-generated responses, and is therefore useful for employing the task in larger samples. However, the current format could be easily adapted to include a free response component, which may provide additional information regarding participants' initial interpretations in the absence of experimenter-generated information. 


\subsubsection{Functional Disability Inventory}

Patients' functional disability was assessed with the Functional Disability Inventory (FDI). This instrument assesses perceived difficulty in performing common activities in the domains of school, home, recreation, and social interactions. Children rate the difficulty they had in carrying out each activity in the preceding two weeks $(0=$ no trouble; $1=$ a little trouble; $2=$ some trouble; $3=$ a lot of trouble; and $4=$ impossible). The FDI consists of 15 items and yields a total score that can range from 0 to 60 , with higher scores indicating greater disability. The FDI has yielded good reliability and validity for children and adolescents [66] and for youth with chronic pain [26]. Cronbach's alpha in this study was .90 for the total score.

\subsubsection{Pain severity indices}

To assess severity of chronic pain, patients completed one 11-point visual analogue scale indicating their average level of pain in the last 3 months $(0=$ no pain; $10=$ worst pain possible), and one 6-point visual analogue scale indicating their frequency of pain in the last 3 months $(1=$ on less than one day each month; 6 = every day). These items were taken from the Brief Pain Inventory [8], which has been widely used to measure pain experiences in clinical and non-clinical populations.

\subsubsection{Pain Catastrophizing Scale-Child version}

Participants' catastrophic thinking about pain was assessed with the Pain Catastrophizing Scale - Child version [11], which was adapted from the adult Pain Catastrophizing Scale [57]. It consists of 13 items that yield a total score from 0 to 52 . Higher scores indicate more pain catastrophizing. Subscale scores for rumination, magnification, and helplessness can be derived. The total score was used for the current study. The PCS-C has good reliability and 
validity for children above 9 years [11]. Cronbach's alpha in this study was .88 for the total score.

\subsubsection{Revised Child Anxiety and Depression Scale}

To assess whether differences in interpretations between adolescents with and without chronic pain were due to differences in generalized anxious or depressive symptomatology, we administered the Revised Child Anxiety and Depression Scale (RCADS [7]). The RCADS consists of 47 items, scored on a 4-point Likert scale from never to always. Higher scores indicate more anxiety or depression. The RCADS comprises six subscales but in this study we only calculated scores for Generalized Anxiety Disorder and Major Depressive Disorder. The RCADS has yielded good reliability and validity for children and adolescents $[6,20,36]$. Cronbach's alpha in this study was .86 for the GAD subscale and .86 for the MDD subscale.

\subsection{Procedure}

For the school sample (control group), all testing sessions took place at school. For the patient sample, all testing sessions took place at the Nuffield Orthopaedic Centre. After completing the consent/assent forms, participants were seated in front of a computer at a distance of approximately $60 \mathrm{~cm}$ from the screen, to complete the AIBT task. Participants completed two practice trials and the experimenter gave additional verbal instructions for any participants who were unclear. After finishing the AIBT task, participants completed the questionnaire measures. Participants in the patient group then began the ABM training protocol (to be described elsewhere). Upon completion, all participants were debriefed as to the nature of the studies. The current study procedure took approximately 45-60 minutes.

\subsection{Data analysis plan}


The AIBT task provides two types of data: 1) ratings of interpretations (i.e., participants' ratings of the different interpretations, in terms of whether they come to mind and their believability, on a scale of 1-5), and 2) forced choice of interpretation (i.e., participants' choice of one interpretation, in terms of whether they come to mind and their believability, for each situation). The ratings data allow us to consider more subtle quantitative differences between individuals in the endorsement of benign and negative interpretations independently, which cannot necessarily be detected with the forced choice data. Thus following previous reports [22], we present only the ratings data here. Indeed, inspection of forced choice data reveals broader main effects of group but no interactions with context or block, supporting these assumptions (forced choice data available on request).

\subsubsection{Group comparisons}

To examine whether interpretations of ambiguous situations differed between adolescents with and without chronic pain (i.e., patient group vs. control group), we performed a multivariate repeated-measures ANOVA. Specifically, we used a 2 × 2 × 2 repeated-measures design with valence (negative/benign word endings), context (bodily threat/social), and block (interpretation generation/belief), as within-subject factors, and group (chronic pain/controls) as a between-subjects factor. A repeated-measures ANOVA was chosen because we were interested in whether the effect of group on both negative and benign interpretations depended on whether participants reported on interpretations coming to mind (interpretation generation) or their belief in those interpretations being true (interpretation belief) (i.e., block), and whether interpretations varied across context (bodily threat/social situations). Performing a single analysis to investigate these questions afforded the most stringent approach to reduce error from multiple comparisons. If a four-way interaction was found, we first decomposed this interaction to investigate the presence of three-way interactions separately for bodily 
threat and social items. We chose to decompose four-way interactions this way (i.e., by context) because we hypothesised that chronic pain patients would show biased interpretations specifically for pain and bodily threat information, rather than for social information, whereas all other comparisons (i.e., Valence: negative, benign; Block: generation, belief) were exploratory. Significant findings were subsequently followed by Bonferroni-corrected analyses of simple main effects.

Participants in the patient and control groups differed significantly in age and gender (see section 3.1.2). Thus, if group differences in AIBT task indices were found, we investigated whether age and gender could explain these group differences by exploring whether age and gender were associated with those AIBT task indices. ANOVAs would also be re-run including gender and age as covariates. In addition, if group differences in the AIBT task were found, we investigated whether these differences could be explained by differences in mood by examining whether anxious and depressive symptomatology was associated with the implicated AIBT task indices. ANOVAs would also again be re-run including anxiety and depressive symptomatology as covariates.

\subsubsection{Associations with disability}

If group differences were identified for any of the AIBT task indices, analyses were then performed to examine whether these indices were linearly associated with disability for adolescents with chronic pain, and whether they could explain unique variance in disability beyond what is explained by measures of pain severity (pain intensity and frequency in the preceding 3 months) and pain catastrophizing. To do so, we performed hierarchical regression analyses with the relevant AIBT task index entered in the first step, and pain intensity, frequency, and catastrophizing score entered in the second step. Functional disability (FDI) 
was entered as the dependent variable. FDI data was collected only from adolescents with chronic pain, so we performed this analysis only for the patient sample $(N=66)$.

\subsubsection{Significance testing}

For all analyses, $p<.05$ was the cut-off for statistical significance, but exact $p$ values are reported in the text to aid critical interpretation of the data. For the ANOVA analyses, Partial Eta Squared $\left(\eta_{p}^{2}\right)$ effect sizes are reported (small effect size $=0.01$; medium effect size $=$ 0.06 ; large effect size $=0.14[9,44])$. Where assumptions for homogeneity of variance are not met, we report statistics for equal variances not assumed.

\section{Results}

\subsection{Participant characteristics}

\subsubsection{Patient group demographics}

Adolescents with chronic pain were predominantly Caucasian $(N=61,92.4 \%)$ and born in the United Kingdom $(N=62,93.9 \%)$. The majority of adolescents had other siblings $(N=59$, 89.4\%). Most adolescents were attending school full time or had completed schooling $(N=$ $53,80.3 \%$ ), although a substantial minority were attending school only part time or receiving home or hospital schooling $(N=12,18.2 \%)$. Time since pain onset varied between 5 and 170 months $(M=45.7$ months). Fifty-eight $(87.9 \%)$ adolescents presented with widespread musculoskeletal pain, whilst eight $(12.1 \%)$ presented with pain in only one body location. Most common pain problems included joint pain $(N=36,54.5 \%)$, pain in legs or feet $(N=35$, $53 \%)$, back pain $(N=33,50 \%)$, and pain in hands or arms $(N=30,45.5 \%)$. Twelve participants $(18.2 \%)$ reported pain all over their body. When asked to indicate the body location where they experienced the most pain, participants most often indicated back pain $(N$ 
$=16,24.2 \%)$, pain in legs or feet $(N=15,22.7 \%)$, or joint pain $(N=10,15.2 \%)$. Pain onset was reported as gradual for 39 adolescents (59.1\%) and sudden for 27 adolescents (40.9\%). Adolescents reported a substantial amount of pain (average pain intensity in the last 3 months: $M=6.55$ out of $10, S D=1.63$ ) and disability (as indexed by Functional Disability Inventory (FDI), $M=22.79, S D=10.74)$. Forty-four adolescents $(66.7 \%)$ reported experiencing pain every day in the last three months, $20(30.3 \%)$ reported pain on most days, and two (3\%) reported pain on about one day per week. Thirty-one (47\%) adolescents reported that they had taken pain medication at some point in the weeks leading up to the study.

\subsubsection{Demographic group comparisons}

Adolescents with and without chronic pain reported similar levels of generalised anxiety (RCADS GAD subscale, Patients: $M=6.94, S D=4.25$; Controls: $M=7.70, S D=3.69 ; t(138)$ $=1.13, p=.26)$ and depressive symptomatology (RCADS Depression subscale, Patients: $M=$ 11.74, $S D=5.98$, Controls: $M=9.99, S D=5.99 ;(t(138)=-1.73, p=.09)$. There were significant differences between adolescents with and without chronic pain in age (Patients: $M$ $=13.97, S D=2.13)$; Controls: $M=14.95, S D=1.71 ; t(124.38)=2.96, p=.004)$ and gender (Patients: 55 (83.3\%) females; Controls: 42 (56.8\%) females; $\left.\chi^{2}(1)=11.58, p=.001\right)$ (see Section 2.4.1 for details on how we controlled for these differences in statistical analyses). Of note, due to a technical fault, data on age was not recorded for one participant in the control sample. Thus, descriptive statistics of age do not include this participant.

\subsection{AIBT task results}

To examine whether adolescents with and without chronic pain differed in their interpretations of ambiguous situations, a 2 (valence) x 2 (block) x 2 (context) ANOVA was conducted with group (chronic pain vs. controls) as between-subjects factor. The multivariate 
test yielded main significant effects for valence, $F(1,138)=10.32, p=.002, \eta_{p}^{2}=.07$; context, $F(1,138)=118.11, p<.001, \eta_{p}^{2}=.46$; and block, $F(1,138)=42.56, p<.001, \eta_{p}^{2}=$ .24. There were also significant two-way interactions for context $\mathbf{x}$ group, $F(1,138)=5.43, p$ $=.02, \eta_{p}^{2}=.04$, for context $\mathbf{x}$ block, $F(1,138)=8.93, p=.003, \eta_{p}^{2}=.06$, and for block $\mathbf{x}$ valence, $F(1,138)=80.14, p<.001, \eta_{p}^{2}=.37$, as well as a significant three-way interaction for valence $\mathbf{x}$ block x context, $F(1,138)=10.60, p=.001, \eta_{p}^{2}=.07$

Of greatest relevance for our hypotheses, there were also significant interaction effects with group. Specifically, there was a significant two-way interaction for valence $\mathbf{x}$ group, $F(1,138)$ $=4.53, p=.04, \eta_{p}^{2}=.03$, and a significant four-way interaction for valence $\mathbf{x}$ block $\mathbf{x}$ context x group, $F(1,138)=6.39, p=.01, \eta_{p}^{2}=.04$. All other interactions failed to reach significance (see Table S1 in supplementary materials for full description of all main and interaction effects).

To decompose the four-way valence $\mathbf{x}$ block $\mathbf{x}$ context $\mathbf{x}$ group interaction, which subsumed all other interactions, we first performed 2 (block) x 2 (valence) ANOVAs with group (chronic pain vs. controls) as between-subjects factor separately for bodily-threat items and social items (see Section 2.4.1 for details). These analyses revealed a valence $\mathbf{x}$ block $\mathbf{x}$ group interaction for bodily threat items $F(1,138)=4.91, p=.03, \eta_{p}^{2}=.03$, but not for social items $F(1,138)=0.89, p=.35, \eta_{p}^{2}=.01$. Results can be seen in Figure 1. Again, all other interactions failed to reach significance (see Table S2 in supplementary materials for full description of all main and interaction effects using separate bodily threat and social analyses). 
Thus, Bonferroni-corrected analyses of simple main effects were performed for the bodily threat items only, with group (chronic pain vs. controls) as the focus for comparison. These analyses revealed that adolescents with chronic pain were significantly more likely to reject benign interpretations of bodily threat situations when reporting on belief in those interpretations than adolescents without chronic pain $(p=.011)$. There were no significant group differences when reporting on whether interpretations came to mind (negative interpretations: $p=.40$; benign interpretations: $p=.16$ ), or for negative interpretations when reporting on belief in those interpretations $(p=.09)$.

\section{[INSERT FIGURE 1 HERE]}

To examine whether group differences in benign belief items could be explained by group differences in age and gender, we first performed Pearson or point-biserial correlations between age and gender (respectively) and the benign belief items. These analyses revealed no associations between benign belief items and gender $(r=.02, p=.85)$ or age $(r=.16, p=$ .06), suggesting that group differences in age and gender cannot explain differences in interpretations. We also performed an additional repeated-measures ANOVA as described above, but including age and gender as covariates. In this analysis, the significant four-way valence $\mathbf{x}$ block $\mathbf{x}$ context $\mathbf{x}$ group interaction remained significant $(F(1,135)=7.09, p=$ $\left..009, \eta_{p}^{2}=.05\right)$, again suggesting that group differences in age and gender did not drive effects.

Finally, similar to previous studies $[22,50]$, we examined whether group differences in the benign belief items could be explained by anxiety and depressive symptomatology by performing Pearson correlations between anxiety and depression scores and the benign belief items. Again, these analyses revealed no associations between benign belief items and anxiety $(r=.02, p=.84)$ or depressive $(r=-.08, p=.36)$ symptomatology. Of note, though, there 
were significant associations between the AIBT task social items and anxiety and depressive symptomatology (see Table S3 in supplementary materials for correlation table), in line with previous findings from the psychopathology literature $[10,16]$. In addition, when performing the repeated-measures ANOVA described above again, but including anxiety and depression scores as covariates, the significant four-way valence $\mathbf{x}$ block $\mathbf{x}$ context $\mathbf{x}$ group interaction remained significant $\left(F(1,136)=6.59, p=.011, \eta_{p}^{2}=.05\right)$, again suggesting that AIBT task differences in interpretations between adolescents with and without chronic pain cannot be explained by differences in anxiety and depressive symptomatology.

\subsection{Associations with disability}

Given that group differences on the AIBT task were found, specifically for the belief in benign bodily threat items, we next examined whether this interpretation index was linearly associated with levels of functional disability in the patient sample. We also examined whether this interpretation index explained unique variance in functional disability beyond what is explained by other important factors that have been previously associated with disability; pain intensity, frequency, and catastrophizing. To do so, we conducted a hierarchical multiple regression analysis with belief in benign bodily threat interpretations entered in the first block, and pain intensity, frequency, and catastrophizing scores entered in the second block. Results for the regression analysis are reported in Table 1. Specifically, patient's belief in benign bodily threat interpretations explained a significant amount of the variance in patients' levels of functional disability $\left(F(1,64)=5.78, p=.019, R^{2}=.08\right)$. Adding pain intensity, frequency, and catastrophizing (PCS) scores further improved the model $\left(F(4,61)=9.12, p<.001 ; R^{2}=.37, p<.001\right)$, however, patients' belief in benign bodily threat interpretations remained a significant predictor of functional disability. Specifically, in the model including all variables, both pain intensity $($ Beta $=.44, t(61)=4.12$, 
$p<.001)$ and belief in benign bodily threat interpretations (Beta $=-.26, t(61)=-2.51, p=.02)$ significantly predicted levels of functional disability, whilst pain catastrophizing score (Beta $=.19, t(61)=1.77, p=.08)$, and pain frequency $($ Beta $=.06, t(61)=0.53, p=.60)$ were nonsignificant. This suggests that the index of belief in benign bodily threat interpretations explains unique variance in patients' levels of functional disability even when controlling for severity of chronic pain and pain catastrophizing. Table S4 in supplementary materials also presents simple Pearson correlations between the pain factors linked to disability and AIBT bodily threat indices.

\section{[INSERT TABLE 1 HERE]}

\section{Discussion}

This study employed the Adolescent Interpretations of Bodily Threat (AIBT) task to compare interpretations of ambiguous information between adolescents with and without chronic pain. In the task, vignettes describing ambiguous situations that could be interpreted as signifying pain and bodily threat, and those that could be interpreted as negative social situations, are presented. As predicted, adolescents with chronic pain endorsed benign (i.e., non-threatening) interpretations of ambiguous bodily-threat situations less than adolescents without chronic pain, particularly when reporting on their belief in those interpretations being true. These differences in interpretational style were not evident for social situations, and not explained by differences in anxious or depressive symptoms. Weaker endorsement of benign interpretations was also associated with more functional disability even after controlling for chronic pain severity (indexed by pain intensity and frequency in the preceding three months), and pain catastrophizing. These results suggest that interpretations of ambiguity over situations regarding pain and bodily threat varied between adolescents with and without 
chronic pain, and may be clinically relevant for understanding levels of functioning among adolescent pain patients.

Our findings are consistent with adult studies, which have demonstrated differences between pain patients and controls on a range of interpretation bias tasks. These tasks have typically assessed responses to single word stimuli such as homographs and homophones (e.g., pain/pane, terminal) [50,51], incomplete word stems [19], or ambiguous facial expressions [30], rather than to more realistic situations as assessed by the AIBT, or other vignette-based tasks used in community adult samples [27,28,61,62]. The AIBT task is also useful for distinguishing whether pain-associated differences arise because of higher ratings of negative interpretations or lower ratings of benign interpretations. Our findings suggesting differences only in the endorsement of benign, but not negative interpretations are somewhat out of line with adult studies, where chronic pain patients are more likely to interpret ambiguous words and faces in a negative, threat-related way [53]. This difference may be due to age differences between studies or differences in task parameters. For example, whilst the AIBT task allows for ratings that span a range of certainty, previous studies using single word stimuli have typically measured only which interpretation first comes to mind (e.g., [50,51]), which may produce more extreme results. Nonetheless, as these findings around benign interpretations are also in line with our previous study employing the AIBT task in a community adolescent sample, they could suggest that interventions encouraging stronger belief in benign interpretations could be potent for adolescent pain patients.

Our findings also extend understanding of cognitive factors in pediatric pain. Previous studies have indicated that youth with chronic pain are characterised by biases in the way they attend to $[3,4,24,63]$ and remember [32] pain-related information. We present here evidence that 
biased interpretations of ambiguous bodily-threat information are also relevant for the experience of chronic pain in youth. Going forward, it will be important to investigate the role of development in the manifestation and influence of interpretation and appraisal processes across childhood and adolescence. For example, it is interesting that in the current study, group differences emerged only when adolescents reported on their belief in interpretations being true (interpretation belief), rather than whether those interpretations came to mind (interpretation generation). This indicates that adolescents do have the cognitive capacity to differentiate their own interpretations from reality. Future studies could investigate whether changes in cognitive capacity across childhood, adolescence, and adulthood are associated with changes in pain-relevant interpretation processes. It will also be interesting to investigate whether similar findings emerge when adolescents are presented with additional contextual cues that may increase or reduce their belief in their initial interpretations.

The current findings are also relevant for the broader literature on cognitive biases and chronic pain. Most work to date has focused on biased attending to pain-related information. In particular, studies highlight the importance of hypervigilance and selective attention for pain $[15,23,65]$, interruption by pain $[2,17]$, and difficulty disengaging from pain $[17,29]$. Yet, in recent theoretical models, biased attending is proposed as an outcome of biased interpretation, specifically, in the interpretation and appraisal of pain and pain-related information as threatening. For example, the Misdirected Problem-Solving Model [18] suggests that the belief that pain is harmful and requires a solution will increase the likelihood that patients will classify ambiguous stimuli as pain- and threat-related, and this interpretation bias will in turn enhance attentional capture and interruption by pain, driving avoidance of activities, and thus disability. More recently, Todd and colleagues synthesized prospective and intervention studies to develop the Threat Interpretation Model [59]. This model proposes 
that the classification of stimuli as pain- and threat-relevant will lead to a pattern of attentional vigilance-avoidance. Thus, recent reconceptualizations suggest that biases in interpretation may in fact drive biased attending, and may therefore be a more relevant target for intervention [12,53]. Relatedly, there already exist novel cognitive training tools that use simple learning mechanisms to encourage more negative or benign interpretations of ambiguous situations [33,35,52], and these tools have recently been applied to an experimental pain setting [25].

This study has various limitations. First, the cross-sectional nature prevents causal hypotheses from being tested. Frequency effects could explain our findings, that is, chronic pain patients may demonstrate different interpretations of ambiguity simply because of their repeated experiences with bodily pain, poor health, and medical settings. However, if this were the case, we would expect group differences in the endorsement of negative interpretations as well as benign interpretations. Nonetheless, longitudinal studies will be necessary for assessing whether or not interpretations causally predict pain chronicity. It will also be useful to recruit additional control groups, for example adolescents who are tested in a medical setting but who do not have pain. Second, although our findings remained significant when controlling for anxious and depressive symptoms, it is interesting that our groups did not differ on these measures. Previous studies typically report elevated levels of anxiety and depression in chronic pain samples [60], and our findings remain to be replicated in other clinical and community samples where mood differences may emerge. Also, our two groups differed on age and sex, and whilst analyses indicated that these factors do not explain current findings, replications with matched samples, in particular from other research groups, are warranted. Third, the patient group were recruited for the purposes of the current study as well as for an intervention study (data not yet reported), which could affect expression of 
interpretation biases, although it is difficult to know whether this contextual difference would lead to over- or under-estimation of biases.

In addition to these limitations, given that the AIBT task items are broadly associated with ill health and bodily threat, we cannot suggest if our findings are reflective of a more specific sensory processing bias in which mild sensations, or ambiguous sensations, are interpreted as painful $[47,50]$. Indeed, this sensory processing bias is most strongly implicated in cognitiveaffective models of chronic pain, and may be most relevant for understanding pain chronicity and maintenance. On the other hand, recent models (e.g., [40]) have emphasised that broader appraisals of bodily threat are also important in chronic pain. Further studies on the specificity of interpretation bias in contributing to chronic pain will be fruitful for comparing and contrasting these models. In addition, whilst our hypothesis that biases would be specific for bodily threat and not social items was driven by cognitive models of pain, previous research has shown that some chronic pain patients report social difficulties as a consequence of their pain $[55,56]$. The social impact of chronic pain, especially for peer interactions, may be especially relevant in adolescence, in which individuals undergo a process of "social reorientation" entailing increased affiliation with peers, relative to family members $[34,41]$. On this basis, we may expect to see interpretation biases that extend to ambiguous social situations in adolescent patients who have experienced negative social consequences of their pain, and future studies should investigate the role of social factors in bias specificity. In addition, we measure interpretation 'generation' by asking participants whether an experimenter-generated interpretation came to mind. Future studies using the AIBT task could include a free response component to better measure self-generation of interpretations. Finally, it is interesting that the benign bodily threat interpretation index was linearly associated with functional disability but not pain indices in the patient sample (see Table S4 
in supplementary materials). Findings require replication and extension in larger samples to disentangle the role of pain-related interpretations in patients' pain experiences and painrelated functioning.

Adolescents with chronic pain, compared with healthy controls, were less likely to endorse benign interpretations of ambiguous bodily-threat information, particularly when reporting on their belief in those interpretations being true. This interpretation pattern was associated with more disability among adolescent patients, even after controlling for severity of chronic pain and pain catastrophizing. The current findings extend our understanding of the role and nature of cognition in adolescent pain, and provide justification for employing the AIBT task in longitudinal and training studies to further investigate causal associations.

\section{Conflict of interest statement}

The authors have no conflicts of interest to declare.

\section{References}

[1] Asmundson GJG, Noel M, Petter M, Parkerson HA. Pediatric fear-avoidance model of chronic pain: Foundation, application and future directions. Pain Res. Manag. 2012;17:397-405.

[2] Attridge N, Crombez G, Van Ryckeghem D, Keogh E, Eccleston C. The Experience of Cognitive Intrusion of Pain. 2015 doi:10.1097/j.pain.0000000000000257.

[3] Beck JE, Lipani T a., Baber KF, Dufton L, Garber J, Smith C a., Walker LS. Attentional bias to pain and social threat in pediatric patients with functional abdominal pain and pain-free youth before and after performance evaluation. Pain 
2011;152:1061-1067. doi:10.1016/j.pain.2011.01.029.

[4] Boyer MC, Compas BE, Stanger C, Colletti RB, Konik BS, Morrow SB, Thomsen AH. Attentional biases to pain and social threat in children with recurrent abdominal pain. J. Pediatr. Psychol. 2006;31:209-220.

[5] Castillo MD, Leandro PG. Interpretation bias in anxiety a synthesis of studies with children and adolescents. Procedia - Soc. Behav. Sci. 2010;5:1105-1111.

[6] Chorpita BF, Moffitt CE, Gray J. Psychometric properties of the Revised Child Anxiety and Depression Scale in a clinical sample. Behav. Res. Ther. 2005;43:309322.

[7] Chorpita BF, Yim L, Moffitt C, Umemoto L a., Francis SE. Assessment of symptoms of DSM-IV anxiety and depression in children: A revised child anxiety and depression scale. Behav. Res. Ther. 2000;38:835-855.

[8] Cleeland CS, Ryan KM. Pain assessment: global use of the Brief Pain Inventory. Ann. Acad. Med. Singapore 1994;23:129-138.

[9] Cohen J. Statistical power analysis for the behavioral sciences. 1988 doi:10.1234/12345678.

[10] Creswell C, Schniering CA, Rapee RM. Threat interpretation in anxious children and their mothers: Comparison with nonclinical children and the effects of treatment. Behav. Res. Ther. 2005;43:1375-1381.

[11] Crombez G, Bijttebier P, Eccleston C, Mascagni T, Mertens G, Goubert L, Verstraeten $\mathrm{K}$. The child version of the pain catastrophizing scale (PCS-C): A preliminary validation. Pain 2003;104:639-646.

[12] Crombez G, Heathcote LC, Fox E. The puzzle of attentional bias to pain : beyond attention. Pain 2015;156:1581-1582. 
[13] Crombez G, Van Ryckeghem DML, Eccleston C, Van Damme S. Attentional bias to pain-related information: A meta-analysis. Pain 2013;154:497-510.

[14] Van Damme S, Legrain V, Vogt J, Crombez G. Keeping pain in mind: A motivational account of attention to pain. Neurosci. Biobehav. Rev. 2010;34:204-213.

[15] Dehghani M, Sharpe L, Nicholas MK. Selective attention to pain-related information in chronic musculoskeletal pain patients. Pain 2003;105:37-46.

[16] Dineen KA, Hadwin JA. Anxious and depressive symptoms and children's judgements of their own and others' interpretation of ambiguous social scenarios. J. Anxiety Disord. 2004;18:499-513.

[17] Eccleston C, Crombez G. Pain demands attention: a cognitive-affective model of the interruptive function of pain. Psychol Bull 1999;125:356-366. doi:10.1037/00332909.125.3.356.

[18] Eccleston C, Crombez G. Worry and chronic pain: A misdirected problem solving model. Pain 2007;132:233-236.

[19] Edwards LC, Pearce S a. Word completion in chronic pain: evidence for schematic representation of pain? J. Abnorm. Psychol. 1994;103:379-382.

[20] Esbjørn BH, Sømhovd MJ, Turnstedt C, Reinholdt-Dunne ML. Assessing the revised child anxiety and depression scale (RCADS) in a national sample of Danish youth aged 8-16 years. PLoS One 2012;7.

[21] Hadwin J, Frost S, French CC, Richards a. Cognitive processing and trait anxiety in typically developing children: evidence for an interpretation bias. J. Abnorm. Psychol. $1997 ; 106: 486-490$.

[22] Heathcote LC, Koopmans M, Eccleston C, Fox E, Jacobs K, Wilkinson N, Lau JYF. Negative interpretation bias and the experience of pain in adolescents. J. Pain 2016. 
doi:10.1016/j.jpain.2016.05.009.

[23] Heathcote LC, Vervoort T, Eccleston C, Fox E, Jacobs K, Van Ryckeghem DML, Lau JYF. The relationship between adolescents' pain catastrophizing and attention bias to pain faces is moderated by attention control. Pain 2015;156:1334-1341.

[24] Hermann C, Zohsel K, Hohmeister J, Flor H. Cortical correlates of an attentional bias to painful and innocuous somatic stimuli in children with recurrent abdominal pain. Pain 2008;136:397-406.

[25] Jones EB, Sharpe L. The effect of cognitive bias modification for interpretation on avoidance of pain during an acute experimental pain task. Pain 2014;155:1569-1576. doi:10.1016/j.pain.2014.05.003.

[26] Kashikar-Zuck S, Flowers SR, Claar RL, Guite JW, Logan DE, Lynch-Jordan AM, Palermo TM, Wilson AC. Clinical utility and validity of the Functional Disability Inventory (FDI) among a multicenter sample of youth with chronic pain. Pain 2011;152:1600-1607. doi:10.1016/j.pain.2011.02.050.

[27] Keogh E, Cochrane M. Anxiety sensitivity, cognitive biases, and the experience of pain. J. Pain 2002;3:320-329.

[28] Keogh E, Hamid R, Hamid S, Ellery D. Investigating the effect of anxiety sensitivity, gender and negative interpretative bias on the perception of chest pain. Pain 2004;111:209-217.

[29] Khatibi A, Dehghani M, Sharpe L, Asmundson GJG, Pouretemad H. Selective attention towards painful faces among chronic pain patients: Evidence from a modified version of the dot-probe. Pain 2009;142:42-47. doi:10.1016/j.pain.2008.11.020.

[30] Khatibi A, Sharpe L, Jafari H, Gholami S, Dehghani M. Interpretation biases in chronic pain patients: an incidental learning task. Eur. J. Pain 2015. 
[31] King S, Chambers CT, Huguet A, MacNevin RC, McGrath PJ, Parker L, MacDonald AJ. The epidemiology of chronic pain in children and adolescents revisited: A systematic review. Pain 2011;152:2729-2738. doi:10.1016/j.pain.2011.07.016.

[32] Koutantji M, Pearce S a., Oakley D a., Feinmann C. Children in pain: An investigation of selective memory for pain and psychological adjustment. Pain 1999;81:237-244.

[33] Lau JYF. Cognitive bias modification of interpretations: A viable treatment for child and adolescent anxiety? Behav. Res. Ther. 2013;51:614-622. doi:10.1016/j.brat.2013.07.001.

[34] De Lorme K, Bell MR, Sisk CL. The Teenage Brain: Social Reorientation and the Adolescent Brain--The Role of Gonadal Hormones in the Male Syrian Hamster. Curr. Dir. Psychol. Sci. 2013;22:128-133. doi:10.1177/0963721413479607.

[35] MacLeod C. Cognitive bias modification procedures in the management of mental disorders. Curr. Opin. Psychiatry 2012;25:114-120.

[36] Mathyssek CM, Olino TM, Hartman CA, Ormel J, Verhulst FC, Van Oort FVA. Does the Revised Child Anxiety and Depression Scale (RCADS) measure anxiety symptoms consistently across adolescence? The TRAILS study. Int. J. Methods Psychiatr. Res. $2013 ; 22: 27-35$.

[37] McKellar JD, Clark ME, Shriner J. The cognitive specificity of associative responses in patients with chronic pain. Br. J. Clin. Psychol. 2003;42:27-39.

[38] Merskey H, Bogduk (Eds) N. Classification of Chronic Pain. Descriptions of Chronic Pain Syndromes and Definitions of Pain Terms. IASP Press 1994:i-xvi.

[39] Miers AC, Blöte AW, Bögels SM, Westenberg PM. Interpretation bias and social anxiety in adolescents. J. Anxiety Disord. 2008;22:1462-1471.

[40] Moseley GL. Reconceptualising pain according to modern pain science. Phys. Ther. 
Rev. 2007;12:169-178.

[41] Nelson EE, Leibenluft E, McClure EB, Pine DS. The social re-orientation of adolescence: a neuroscience perspective on the process and its relation to psychopathology. Psychol. Med. 2005;35:163-174.

[42] Noel M, Palermo TM, Chambers CT, Taddio A, Hermann C. Remembering the pain of childhood : applying a developmental perspective to the study of pain memories. $2015 ; 156$.

[43] Noel M, Rabbitts JA, Tai GG, Palermo TM. Remembering pain after surgery: a longitudinal examination of the role of pain catastrophizing in children's and parents' recall. Pain 2015;156:800-8. doi:10.1097/j.pain.0000000000000102.

[44] Olejnik S, Algina J. Measures of Effect Size for Comparative Studies: Applications, Interpretations, and Limitations. Contemp. Educ. Psychol. 2000;25:241-286. doi:10.1006/ceps.2000.1040.

[45] Orchard F, Pass L, Reynolds S. 'It Was All My Fault'; Negative Interpretation Bias in Depressed Adolescents. J. Abnorm. Child Psychol. 2016;44:991-998.

[46] Orchard F, Pass L, Reynolds S. Associations Between Interpretation Bias and Depression in Adolescents. Cognit. Ther. Res. 2016:1-7.

[47] Pennebaker JW. The Psychology of Physical Symptoms. New York: Springer-Verlag, 1982.

[48] Perquin CW, Hazebroek-Kampschreur A a JM, Hunfeld J a M, Bohnen AM, Van Suijlekom-Smit LW a, Passchier J, Van Der Wouden JC. Pain in children and adolescents: A common experience. Pain 2000;87:51-58.

[49] Pincus T, Morley S. Cognitive-processing bias in chronic pain: A review and integration. Psychol. Bull. 2001;127:599-617. 
[50] Pincus T, Pearce S, McClelland A, Farley S, Vogel S. Interpretation bias in responses to ambiguous cues in pain patients. J. Psychosom. Res. 1994;38:347-353.

[51] Pincus T, Pearce S, Perrott a. Pain patients' bias in the interpretation of ambiguous homophones. Br. J. Med. Psychol. 1996;69 ( Pt 3):259-266.

[52] Salemink E, Wiers RW. Adolescent threat-related interpretive bias and its modification: The moderating role of regulatory control. Behav. Res. Ther. 2012;50:40-46. doi:10.1016/j.brat.2011.10.006.

[53] Schoth DE, Liossi C. Biased Interpretation of Ambiguous Information in Patients With Chronic Pain: A Systematic Review and Meta-Analysis of Current Studies. Health Psychol. 2016. doi:10.1037/hea0000342.

[54] Schoth DE, Nunes VD, Liossi C. Attentional bias towards pain-related information in chronic pain; a meta-analysis of visual-probe investigations. Clin. Psychol. Rev. $2012 ; 32: 13-25$.

[55] Snelling J. The effect of chronic pain on the family unit. J. Adv. Nurs. 1994;19:543551. doi:10.1111/j.1365-2648.1994.tb01119.x.

[56] Strunin L, Boden LI. Family consequences of chronic back pain. Soc. Sci. Med. 2004;58:1385-1393.

[57] Sullivan MJL, Bishop SR, Pivik J. The Pain Catastrophizing Scale: Development and validation. Psychol. Assess. 1995;7:524-532.

[58] Taghavi MR, Moradi AR, Neshat-Doost HT, Yule W, Dalgleish T. Interpretation of ambiguous emotional information in clinically anxious children and adolescents. Cogn. Emot. 2000;14:809-822.

[59] Todd J, Sharpe L, Johnson A, Nicholson K, Colagiuri B, Dear BF. Towards a new model of attentional biases in the development, maintenance, and management of pain. 
Pain 2015;156:1589-1600.

[60] Tsang A, Von Korff M, Lee S, Alonso J, Karam E, Angermeyer MC, Borges GLG, Bromet EJ, de Girolamo G, de Graaf R, Gureje O, Lepine JP, Haro JM, Levinson D, Oakley Browne MA, Posada-Villa J, Seedat S, Watanabe M. Common Chronic Pain Conditions in Developed and Developing Countries: Gender and Age Differences and Comorbidity With Depression-Anxiety Disorders. J. Pain 2008;9:883-891.

[61] Vancleef LMG, Hanssen MM, Peters ML. Are individual levels of pain anxiety related to negative interpretation bias? An examination using an ambiguous word priming task. Eur. J. Pain (United Kingdom) 2015;20:833-841.

[62] Vancleef LMG, Peters ML. Examining content specificity of negative interpretation biases with the Body Sensations Interpretation Questionnaire (BSIQ). J. Anxiety Disord. 2008;22:401-415.

[63] van der Veek SMC, Derkx BHF, Plak RD, Benninga M a, Boer F, Lindauer RJL, de Haan E. Attentional Bias to Activity of Different Parts of the Body in Children With Functional Abdominal Pain: An Experimental Study. J. Pediatr. Psychol. 2014;39:112. doi:10.1093/jpepsy/jsu004.

[64] Vervoort T, Trost Z, Van Ryckeghem DML. Children's selective attention to pain and avoidance behaviour: The role of child and parental catastrophizing about pain. Pain 2013;154:1979-1988. doi:10.1016/j.pain.2013.05.052.

[65] Vlaeyen JWS, Linton SJ. Fear-avoidance and its consequences in chronic musculoskeletal pain: A state of the art. Pain 2000;85:317-332.

[66] Walker LS, Greene JW. The functional disability inventory: measuring a neglected dimension of child health status. J. Pediatr. Psychol. 1991;16:39-58. 


\section{Figure legends}

Figure 1. AIBT task ratings (error bars $=\mathrm{SE},{ }^{*} p<.05$ )

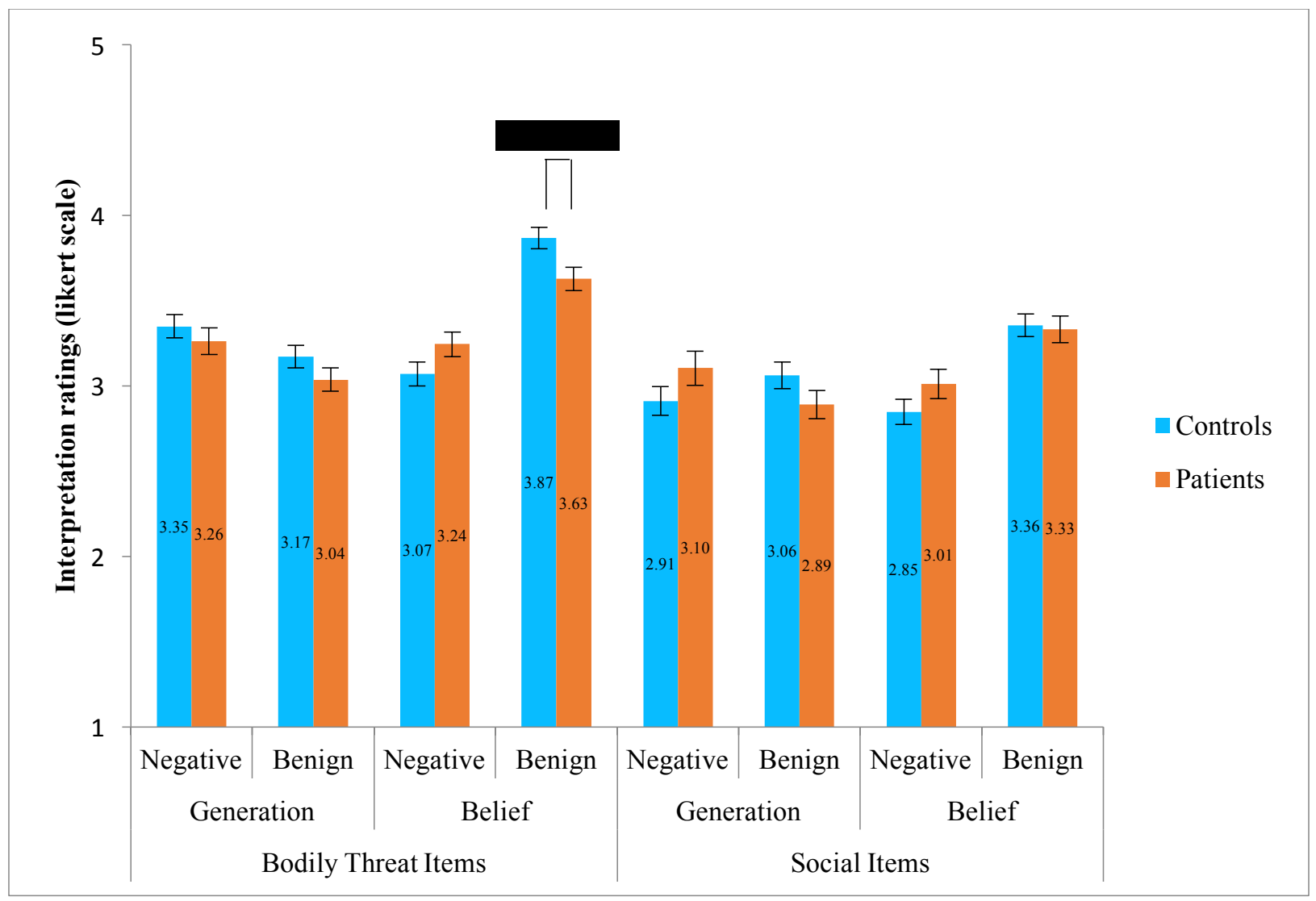


Table 1. Results of hierarchical multiple regression analysis with Functional Disability score (FDI) as the dependent variable

\begin{tabular}{lc|c|c|c}
\hline & & $b$ & $S E b$ & $\beta$ \\
\hline Step 1 & Constant & 42.92 & 8.42 & \\
& Belief in benign bodily threat (AIBT index) & -5.52 & 2.30 & $-.29^{*}$ \\
Step 2 & & & \\
& Celief in benign bodily threat (AIBT index) & -4.96 & 1.98 & $-.26^{*}$ \\
& Pain frequency (last 3 months) & 1.10 & 2.08 & .06 \\
& Pain intensity (last 3 months) & 2.93 & 0.71 & $.44^{* * *}$ \\
\multicolumn{2}{c|}{ Pain Catastrophizing (PCS) } & 0.2 & 0.11 & .19 \\
\hline
\end{tabular}

Note. $R^{2}=.08$ for Step $1(p=.02) . \Delta R^{2}=.29$ for Step $2(p<.001)$.

$*_{p}<.05, * * * p<.001$ 